\title{
Factors associated with psychotic symptoms in Alzheimer's disease
}

Nobutsugu Hirono, Etsuro Mori, Minoru Yasuda, Yoshitaka Ikejiri, Toru Imamura, Tatsuo Shimomura, Manabu Ikeda, Mamoru Hashimoto, Hikari Yamashita

\begin{abstract}
Objectives-Many clinical and biological factors have been reported to be associated with the presence of psychosis in patients with Alzheimer's disease, although the associations were variable. The aim of this study was to clarify factors associated with the presence of psychosis in patients with Alzheimer's disease.

Methods-Psychiatric functioning was studied in 228 patients with Alzheimer's disease based on the results of the behavioural pathology in Alzheimer's disease rating scale or the neuropsychiatric inventory. The effects of sex, education level, age, duration of illness, cognitive function, and apolipoprotein $E$ genotype were investigated for dichotomous psychotic status with a multiple logistic regression analysis.
\end{abstract}

Results-Of the 228 patients with Alzheimer's disease, 118 (51.8\%) showed evidence of delusions or hallucinations. Of these, 94 had delusions only, three had hallucinations only, and 21 had both. Older age, female sex, longer duration of illness, and more severe cognitive impairment were the factors independently associated with the presence of psychosis. The presence of psychosis was not significantly related to either educational level or apolipoprotein $\mathrm{E}$ genotype.

Conclusions-Age, sex, and severity of illness were independent factors associated with the presence of psychosis in patients with Alzheimer's disease. The reason why some patients with Alzheimer's disease develop psychosis remains unclear. There may be distinctive subtypes of Alzheimer's disease or the presence of individual factors which affect the development of psychosis.

(F Neurol Neurosurg Psychiatry 1998;64:648-652)

Keywords: Alzheimer's disease; psychotic symptoms; apolipoprotein E

Psychotic symptoms are common in patients with Alzheimer's disease and have a major impact on their quality of life and social affiliations. In a review of 21 studies, Wragg and Jeste $^{1}$ reported that the prevalence of delusion ranged from $10 \%$ to $73 \%$ (median $33.5 \%$ ) and that of hallucination ranged from $21 \%$ to $49 \%$ (median 28\%) in patients with Alzheimer's disease at some time in the course of their illness. Drevets and Rubin ${ }^{2}$ reported that the prevalence of delusions and hallucinations in patients with Alzheimer's disease ranged from $13 \%$ to $75 \%$ and from $3 \%$ to $50 \%$, respectively. Psychotic symptoms have been shown to be a major cause of anxiety and concern for caregivers $^{34}$ and a frequent cause of admission to an institution. ${ }^{56}$ Because appropriate management of behavioural symptoms may lessen the burden of care givers and may postpone admission to an institution, evaluation and management of psychotic symptoms are of considerable importance in practice.

The underlying mechanism for psychosis in Alzheimer's disease is not well understood. Although psychosis has been reported to be associated with many clinical and biological factors, such as age, ${ }^{7-11} \mathrm{sex}^{,{ }^{1-14}}$ duration of illness ${ }^{15}$ education level, ${ }^{13}$ cognitive impairment, ${ }^{13}{ }^{16-21}$ and apolipoprotein E (ApoE) genotypes, ${ }^{22}$ the associations were variable. These inconsistencies are likely explained by one or more methodological factors: (1) the lack of statistical power with small sample sizes; (2) differing diagnostic criteria of the disease and definition of the psychotic symptoms; and (3) the lack of consideration of interactions among factors. In the present study, we examined a large series of patients with Alzheimer's disease for the presence of psychosis by using standardised assessment instruments of psychopathology for patients with dementia, and multivariate statistics to concurrently analyse the patients' characteristics that are possibly related to psychosis.

\section{Methods}

All procedures of this study strictly followed the clinical study guidelines of the ethics committee of Hyogo Institute for Aging Brain and Cognitive Disorders, Himeji, Japan (1993), and were approved by the internal review board. After a complete description of all procedures of this study, written informed consent was obtained from patients or their relatives.

The subjects of this study were 228 of a consecutive series of 312 Japanese patients with sporadic Alzheimer's disease who were given a short term admission to our hospital for examination between July 1993 and September 1996. All patients were examined by both neurologists and psychiatrists and were given routine laboratory tests and standard neuropsychological examinations. In addition, EEG, brain MRI, MR angiography of the neck and head, and cerebral perfusion/metabolism studies by PET or single photon emission computed tomography (SPECT) were done. All results were incorporated in the diagnosis. The 
inclusion criteria were those of both the Diagnostic and Statistical Manual of Mental Disorders, either Third Edition-Revised (DSM-III$\mathrm{R})^{23}$ or Fourth Edition (DSM-IV) ${ }^{23 a}$ for Dementia of the Alzheimer's Type and the National Institute of Neurological and Communicative Disorders and Stroke-Alzheimer's Disease and Related Disorders Association ${ }^{24}$ for probable Alzheimer's disease. Eighty four patients who were admitted during the study period were not studied because of the exclusion criteria, consisting of complications of other neurological diseases or illness resulting from physical disease, evidence of parkinsonism or focal brain lesions on MRI, the presence of severe hearing or vision impairments, a history of mental illness or substance misuse before the onset of dementia, the absence of reliable informants, such as living with family or care givers, or the inability to obtain informed consent.

The included patients consisted of 173 women and 55 men; the mean (SD) age at examination was $72.7(8.2)$ years and the mean educational attainment was 8.9 (2.2) years. The functional severity was very mild in 30 patients, mild in 119 patients, moderate in 63 patients, and severe in 16 patients as determined by the clinical dementia rating scale. ${ }^{25}$ The mean age at symptom onset and duration of symptoms determined with informant based interviews were 67.1 (7.5) years and 37.3 (28.8) months respectively. The mean value of the mini mental state examination (MMSE) ${ }^{26}$ was 18.5 (5.1). Twenty three patients could not complete the Wechsler adult intelligence scale-revised. ${ }^{27}$ The mean values of the remaining 205 patients were 77.0 (10.4) for verbalIQ, 75.1 (13.7) for performance-IQ, and 74.3 (11.9) for full-IQ. The mean number of words recalled in the 10 word list recall subtest of the Alzheimer's disease assessment scale-cognitive part $^{28}$ was 3.3 (1.6).

According to DSM-IV, ${ }^{23 a}$ delusion was defined as a firmly sustained, false belief based on incorrect inference about external reality not attributable to the patient's cultural or subcultural experience, and hallucination was defined as sensory perception that has the compelling sense of reality of a true perception but that occurs without external stimulation of the relevant sensory organ. With the behavioural pathology in Alzheimer's disease rating scale ${ }^{12}$ or the neuropsychiatric inventory, ${ }^{29}$ a single rater assessed each patient's psychiatric condition during an interview with the care giver. In this study, delusions and hallucinations were rated on a present or absent basis. The patient was rated as psychotic when he or she had either delusions or hallucinations.

The age at onset and the duration of the disease were ascertained through an interview with the primary care giver. Age at onset was defined as the age of the first appearance of symptoms of sufficient severity to interfere with social or occupational functioning and the duration was defined as the time in months between the onset and the admission. ${ }^{30}$

The detailed method for ApoE genotyping is described elsewhere. ${ }^{31}$ In brief, genomic DNA was extracted from whole blood samples by the phenol/chloroform method and was amplified by the polymerase chain reaction (PCR) as described by Wenham et al. ${ }^{32}$ The PCR products were digested with 10 units Hha I for five hours at $37^{\circ} \mathrm{C}$. The DNA fragments were electrophoresed for five hours at $60 \mathrm{~mA}$ through a $15 \%$ non-denaturing polyacrylamide gel. The gel was stained with ethidium bromide and photographed under ultraviolet light. The genotypes were determined by the size of DNA fragments. The patients with one or more ApoE $\varepsilon 4$ alleles were rated as $\varepsilon 4$ positive and the others were rated as $\varepsilon 4$ negative.

We used the $\chi^{2}$ test for binary variables, and the two tailed $t$ test for continuous variables for unadjusted comparisons of patients with and without psychosis. For correlational analyses, we used Pearson correlation coefficients. To examine the specific characteristics that predict psychosis, a multiple logistic analysis was used, with psychosis as a dependent variable and the patients' characteristics (sex, education level, duration of illness, MMSE, ApoE genotype, and either age at onset or age at examination) as independent variables. Because age at examination and age at onset were strongly correlated, which causes multicolinearity, each variable was examined in separate models. All statistical analyses were carried out with SAS Release 6.10 software (SAS Institute Inc).

\section{Results}

The ApoE genotyping was $\varepsilon 2 / 3$ in eight patients, $\varepsilon 2 / 4$ in three patients, $\varepsilon 3 / 3$ in 78 patients, $\varepsilon 3 / 4$ in 115 patients, and $\varepsilon 4 / 4$ in 24 patients. Thus, the ApoE $\varepsilon 4$ gene was positive in 142 patients and negative in 86 patients. As expected, the age at examination was highly correlated with the age at onset $(r=0.96, \mathrm{p}<$ 0.0001). The educational attainment was inversely correlated with both age at examination $(r=-0.31, \mathrm{p}<0.0001)$ and age at onset $(r=-0.32, \quad \mathrm{p}<0.0001)$. Both mean age at examination and age at onset were significantly greater in women than in men (73.3 (SD 7.8) v 70.7 (9.2), $\mathrm{p}=0.046 ; 70.4(8.30)$ v 67.5 (9.37), $\mathrm{p}=0.034)$ ). The mean educational attainment was significantly less in women than in men (8.6 (SD 2.1) $v 9.6(2.3), \mathrm{p}=0.0048)$ ). Although age, education level, and the MMSE score did not differ significantly between the ApoE $\varepsilon 4$ positive patients and the $\varepsilon 4$ negative patients, the mean duration of illness was significantly greater in the $\varepsilon 4$ positive patients than in the $\varepsilon 4$ negative patients (39.0 (SD 30.9) v 30.9 (23.6) months, $\mathrm{p}=0.038$ ).

Of the 228 patients, 118 (51.8\%) were rated as psychotic. Of these 118 patients, 21 had both delusions and hallucinations, 94 had delusions only, and three had hallucinations only. Thus $115(50.4 \%)$ of the patients had delusions. Of these, 91 patients had persecutory delusions ( 88 patients had delusions that "people are stealing things", 10 patients had delusions of "being conspired against or harassed", and three patients had a delusion of abandonment), 58 patients had misidentification delusions (42 patients had delusions that "someone was in the house", 11 patients had 
Table 1 Comparison of characteristics between patients with and without psychosis

\begin{tabular}{lllll}
\hline & $\begin{array}{l}\text { Psychotic } \\
(n=118)\end{array}$ & $\begin{array}{l}\text { Non-psychotic } \\
(n=110)\end{array}$ & Statistical value & p Value \\
\hline Age at examination (y) & $74.5(6.9)$ & $70.7(9.0)$ & $\mathrm{t}(226)=3.50$ & 0.0006 \\
Sex (female : male) & $97: 21$ & $76: 34$ & $\chi^{2}(1)=5.35$ & 0.021 \\
Education (y) & $8.5(2.0)$ & $9.2(2.4)$ & $\mathrm{t}(226)=2.20$ & 0.029 \\
Duration of illness (months) & $39.7(30.7)$ & $31.8(25.7)$ & $\mathrm{t}(226)=2.09$ & 0.037 \\
Age at onset (y) & $71.1(7.7)$ & $68.2(9.3)$ & $\mathrm{t}(226)=2.62$ & 0.009 \\
ApoE \&4 (positive : negative) & $76: 42$ & $66: 44$ & $\chi^{2}(1)=0.47$ & 0.49 \\
MMSE & $18.5(4.2)$ & $19.5 \pm 4.0$ & $\mathrm{t}(226)=2.71$ & 0.007 \\
\hline
\end{tabular}

Mean (SD).

$\mathrm{ApoE}=$ apolipoprotein $\mathrm{E} ; \mathrm{MMSE}=$ mini mental state examination.

delusions that "the house was not their own", six patients had delusions that "television figures are actually present in the home", and six patients had other types of misidentification), three patients had delusional jealousy, one patient had a delusion of poverty, one patient had grandiose delusions, and one patient had eromanic delusions. Some patients had two or more kinds of delusions. Twenty four $(10.5 \%)$ of the patients had hallucinations. Of these, 16 had visual hallucinations, 14 had auditory hallucinations, and one had olfactory hallucinations. Seven patients had two types of hallucinations.

Table 1 compares the characteristics of patients with and without psychosis. Those with the psychosis were significantly older, had an older age of onset, had a longer duration of illness, and had less education than those without psychosis. The proportion of women was significantly higher in the psychotic group than in the non-psychotic group. There were no significant associations between psychosis and ApoE genotype. The mean MMSE score was significantly lower in patients with psychosis than in the those without psychosis.

Table 2 shows the results of the first model of multiple logistic regression in which age at onset was included, and table 3 shows that of the second model including age at examination. Both models indicated that the effects of age, sex, and MMSE were significantly associated with the presence of psychosis. In the first model but not in the second, duration of illness was a significant risk factor for psychosis. The

Table 2 Results of multiple logistic regression analysis with age at onset included

\begin{tabular}{llllll}
\hline & Odds ratio & $95 \%$ CI & Wald $\chi^{2}$ & $p$ Value & Effect \\
\hline $\begin{array}{l}\text { Age at onset } \\
\text { Sex }\end{array}$ & 1.73 & $1.19-2.52$ & 8.33 & 0.0039 & $\begin{array}{l}10 \text { year increase } \\
\text { female increase relative to } \\
\text { male }\end{array}$ \\
& 2.01 & $1.03-3.96$ & 4.12 & 0.042 & $\begin{array}{l}\text { 1 year increase } \\
\text { Education }\end{array}$ \\
$\begin{array}{l}\text { Duration of } \\
\quad \text { illness }\end{array}$ & 1.95 & $0.83-1.09$ & 0.58 & 0.45 & \\
$\begin{array}{l}\text { ApoE } \varepsilon 4 \text { positive } \\
\text { MMSE }\end{array}$ & 1.06 & $1.03-1.36$ & 5.71 & 0.017 & 12 month increase \\
& 0.94 & $0.59-1.88$ & 0.03 & 0.86 & $\begin{array}{l}\varepsilon 4+\text { increase relative to } \varepsilon 4- \\
1 \text { point increase }\end{array}$ \\
\hline
\end{tabular}

$\mathrm{CI}=$ confidence interval $; \mathrm{ApoE}=$ apolipoprotein $\mathrm{E} ; \mathrm{MMSE}=$ Mini-Mental State Examination.

Table 3 Results of multiple logistic regression analysis with age at examination included

\begin{tabular}{llllll}
\hline & $\begin{array}{c}\text { Odds } \\
\text { ratio }\end{array}$ & $95 \%$ CI & Wald $\chi^{2}$ & $p$ Value & Effect \\
\hline Age at examination & 1.77 & $1.21-2.57$ & 8.91 & 0.0028 & $\begin{array}{l}10 \text { year increase } \\
\text { female increase relative to } \\
\text { Sex }\end{array}$ \\
& 2.02 & $1.03-3.98$ & 4.15 & 0.042 & male \\
Education & 0.95 & $0.83-1.09$ & 0.53 & 0.47 & 1 year increase \\
Duration of illness & 1.12 & $0.98-1.28$ & 2.88 & 0.09 & 12 month increase \\
ApoE $\varepsilon 4$ positive & 1.05 & $0.59-1.88$ & 0.03 & 0.86 & $\varepsilon 4+$ increase relative to $\varepsilon 4-$ \\
MMSE & 0.94 & $0.88-0.99$ & 4.88 & 0.027 & 1 point increase \\
\hline
\end{tabular}

$\mathrm{CI}=$ confidence interval $; \mathrm{ApoE}=$ apolipoprotein $\mathrm{E} ; \mathrm{MMSE}=$ mini mental state examination. level of education was not a significant predictor of psychosis in either model. The ApoE genotype also had no significant association with psychosis.

\section{Discussion}

The present study provides further evidence that psychotic symptoms are a common manifestation of Alzheimer's disease. The prevalences of delusions $(51.8 \%)$ and hallucinations $(10.5 \%)$ were well within the ranges reported in the literature. ${ }^{12}$ The finding that persecutory delusions (especially the belief that people are stealing or hiding objects) were the most common psychotic manifestations was also compatible with the findings of previous studies.9 ${ }^{12} 1416172033$ Because patients who had parkinsonism were excluded from this study, the chance of erroneous inclusion of those with dementia with Lewy bodies, ${ }^{34}$ in whom neuropsychiatric symptoms are common, was small. The major findings of our study were that sex, age, and severity of cognitive impairment were each significantly and independently associated with psychosis, and that the level of education and the frequency of the ApoE $\varepsilon 4$ allele were not significant predictors of the presence of psychosis.

Previous studies have disagreed on the contribution of cognitive impairment in psychotic symptoms. Some studies failed to show a difference in cognitive impairment between the patients with and without psychosis, ${ }^{7} 10111415{ }^{35-37}$ whereas others showed that psychotic symptoms were associated with poor $^{13}{ }^{17-1921}$ or preserved ${ }^{16}$ cognitive function. These controversies are likely due to the inclusion of patients with different severities of dementia. Psychotic symptoms were considered to be uncommon in both the very mild ${ }^{33}$ and very severe ${ }^{1820}$ stages of Alzheimer's disease. A longitudinal study ${ }^{2}$ disclosed that delusions in patients with Alzheimer's disease were relatively more frequent during the moderate stage. The small proportion of advanced patients included in our study contributed to the positive correlation between psychosis and cognitive impairments. In any case, as the difference in MMSE between groups was small, the effect of cognitive impairment in psychosis would be modest. Nevertheless, it is conceivable that a dysfunction of specific cognitive domains is associated with psychosis.

In this study, duration of illness was shown to be an independent predictor of psychosis in the logistic regression model in which age at onset was the variable, although it was not significant in the model when age at examination was the variable. It is plausible that the effect of duration of illness was masked by the effect of the age at examination, because the age at examination is a function of age at onset and duration of illness. Our results supported the conclusion of the study of Migliorelli et $a l^{15}$ who reported that patients with Alzheimer's disease with delusions had a significantly longer duration of illness than those without delusions, whereas most previous studies failed to show a significant association between duration of illness and psychosis. ${ }^{8} 101318202135-38$ An 
association between development of psychotic symptoms and a rapid mental deterioration has been found in several longitudinal studies. $^{2} 9333940$ This does not disagree with our finding that psychotic patients with Alzheimer's disease had a significantly longer duration of illness, because long duration does not necessarily imply slow progression of the disease. Our findings that a decreased MMSE and a longer duration of illness were separate factors associated with the presence of psychosis would suggest that deterioration of both cognitive and non-cognitive functions promotes psychosis independently.

Older age was shown to be a significant risk factor for psychosis in both logistic regression models in this study. The issue of the effect of age on psychosis in Alzheimer's disease is highly controversial. Several investigators ${ }^{7-11}$ showed that older patients were more likely to have psychotic symptoms, whereas Gilly et al reported that early onset was significantly associated with psychosis when cognitive functions were severely impaired. Devanand et $a l^{13}$ maintained that age at examination was associated with paranoid delusions and inversely correlated with hallucinations. Moreover, many studies failed to show a significant relation between age and psychosis. ${ }^{12} 15$ 17-21 35-38 41 42 However, evidence that supports the relation between older age and development of psychosis is available in studies focusing on localisation of pathological changes in relation to age and psychosis. Senile plaques both in the temporal neocortex and in the hippocampus were more frequent in younger patients with Alzheimer's disease than in elder patients, ${ }^{43} 44$ whereas psychosis in Alzheimer's disease is reportedly inversely related to the preservation in those areas. ${ }^{45}$

We also found a significant association between female sex and development of psychosis. Although female sex has been implicated as a risk factor for Alzheimer's disease, few studies have considered whether sex specifically predisposes patients to the development of psychotic symptoms in Alzheimer's disease. Devanand et $a l^{13}$ reported that women's scores of delusion on the Columbia University scale for psychopathology were significantly higher than those of men. Reisberg et $a l^{12}$ found that women tended to exhibit behavioural symptoms more often. On the other hand, Burns et $a l^{14}$ reported a higher prevalence in men of delusions of theft. Other studies failed to show an association of psychosis with sex. ${ }^{1011} 1520213537384146$ However, in other domains of neurological disease, there is evidence of female sex as a risk factor of psychosis. Comparing psychotic and non-psychotic epileptic patients, Taylor ${ }^{47}$ showed that female sex was a risk factor for development of psychosis. Our finding and those of others remain unexplained, and relevant factors involving genetic traits, as well as cultural and psychosocial involvements, should be investigated.

The multivariate analysis showed that the level of education was not an independent protection factor for psychosis. The association between psychosis and fewer years of education shown in the univariate analysis is likely to be an epiphenomenon which can be accounted for by the inverse correlation between the educational attainment and age and by the negative association between the level of education and female sex. Although one study ${ }^{13}$ reported that education was inversely related to psychosis, the results of our multivariate analysis agreed with most studies, which denied this effect. ${ }^{1011151820213341}$

It has been postulated that patients with Alzheimer's disease with and without the $\varepsilon 4$ allele have distinguishing phenotypic characteristics. ${ }^{42}$ Ramachandran et $a l,{ }^{22}$ in a preliminary report, showed that patients with Alzheimer's disease and the ApoE $\varepsilon 3 / 4$ genotype had more than a threefold increase in psychosis when compared with those with the ApoE $\varepsilon 3 / 3$ genotype. As female patients with late onset Alzheimer's disease carried the $\varepsilon 4$ allele more often than did male patients, ${ }^{31}$ their finding might be attributable to the effects of sex and age. Lehtovirta et $a l,{ }^{42}$ Lopez-Alberola et $a l,{ }^{48}$ and Lopez et $a l^{49}$ showed no association between the ApoE $\varepsilon 4$ gene and development of psychosis. In our study, in which other variables were concurrently examined, this association was clearly denied.

The present study showed that a subpopulation of patients with Alzheimer's disease who are female, of late onset, of severe cognitive impairment, and of long duration of illness, have a high risk for development of psychosis. One possible explanation for our findings is that a particular subtype of Alzheimer's disease which preferentially involves old women has distinctive clinical and neuropathological features. An alternative explanation is that characteristics including genetic, psychosocial, and environmental factors may affect development of psychosis in individuals. Further studies are needed to consider these hypotheses.

We thank Drs Hajime Kitagaki and Kazunari Ishii (Division of Neuroimaging Research) for their help in various parts of the study, and Yoko Takatsuki, Akitsugu Tokimasa, and Misato Fujimori (Neurorehabilitation Service) for their technical assistance.

1 Wragg RE, Jeste DV. Overview of depression and psychosis in Alzheimer's disease. Am F Psychiatry 1989;146:577-87.

2 Drevets WC, Rubin EH. Psychotic symptoms and the longitudinal course of senile dementia of the Alzheimer type. Biol Psychiatry 1989;25:39-48

3 Green JG, Smith R, Gardiner M, et al. Measuring behavioural disturbance of elderly demented patients in the community and its effects on relatives: a factor analytic study. Age Aging 1982;11:121-6.

4 Deimling GT, Bass DM. Symptoms of mental impairment among elderly adults and their effects on family caregivers. f Gerontol $1986 ; 41: 778-84$.

5 Ferris SH, Steinberg G, Shulman E, et al. Institutionalization of Alzheimer's patients: reducing precipitating factors
tion through family counseling. Archives of the Foundation of Thanatology 1985;12:7.

6 Steele C, Rovner B, Chase GA, et al. Psychiatric symptoms and nursing home placement of patients with Alzheimer's disease. Am f Psychiatry 1990;147:1049-51.

7 Swearer JM, Drachman DA, O'Donnell BF, et al. Troublesome and disruptive behaviors in dementia. Relationships to diagnosis and disease severity. $\mathcal{F}$ Am Geriatr Soc 1988;36: 784-90.

8 Gilley DW, Wilson RS, Bennett DA, et al. Predictors of behavioral disturbance in Alzheimer's disease. $\mathcal{F}$ Gerontol 1991;46:362-71.

9 Förstl H, Besthorn C, Geiger-Kabisch C, et al. Psychotic features and the course of Alzheimer's disease: relationship to to cognitive, electroencephalographic and computerized Scand 1993;87:395-9.

10 Binetti G, Padovani A, Magni E, et al. Delusions and dementia: clinical and CT correlates. Acta Neurol Scand 1995;91:271-5 
11 Kotrla KJ, Chacko RC, Harper RG, et al. Clinical variables associated with psychosis in Alzheimer's disease. Am 7 Psyassociated with psychosis
chiatry 1995;152:1377-9.

12 Reisberg B, Borenstein J, Salob SP, et al. Behavioral symptoms in Alzheimer's disease: phenomenology and treatment. F Clin Psychiatry 1987;48(suppl 5):9-15.

13 Devanand DP, Miller L, Richards M, et al. The Columbia University scale for psychopathology in Alzheimer's disease. Arch Neurol 1992;49:371-6.

14 Burns A, Jacoby R, Levy R. Psychiatric phenomena in Alzheimer's disease. I: Disorders of thought content. $\mathrm{Br} F$ Psychiatry 1990;157;72-6.

15 Migliorelli R, Petracca G, Tesón A, et al. Neuropsychiatric and neuropsychological correlates of delusions in Alzheimer's disease. Psychol Med 1995;25:505-13.

16 Cummings JL, Miller B, Hill MA, et al. Neuropsychiatric aspects of multi-infarct dementia and dementia of the Alzheimer type. Arch Neurol 1987;44:389-93.

17 Patterson MB, Schnell AH, Martin RJ, et al. Assessment of behavioral and affective symptoms in Alzheimer's disease. $\mathcal{F}$ Geriatr Psychiatry Neurol 1990;3:21-30.

18 Jeste DV, Wragg RE, Salmon DP, et al. Cognitive deficits of patients with Alzheimer's disease with and without delusions. Am f Psychiatry 1992;149:184-9.

19 Rubin EH, Kinscherf DA, Morris JC. Psychopathology in younger versus older persons with very mild and mild dementia of the Alzheimer type. Am F Psychiatry 1993;150 639-42.

20 Binetti G, Bianchetti A, Padovani A, et al. Delusions in Alzheimer's disease and multi-infarct dementia. Acta Neurol Scand 1993;88:5-9.

21 Doody RS, Massman P, Mahurin R, et al. Positive and negative neuropsychiatric features in Alzheimer's disease. $\mathcal{F}$ Neuropsychiatr Clin Neurosci 1995;7:54-60.

22 Ramachandran G, Marder K, Tang M, et al. A preliminary study of apolipoprotein E genotype and psychiatric manifestations of Alzheimer's disease. Neurology 1996;47: 256-9.

23 American Psychiatric Association: Diagnostic and statistical manual of mental disorders, 3rd ed. Washington DC:APA, 1987.

23a American Psychiatric Association: Diagnostic and statistical manual of mental disorders, 4th ed. Washington, DC: APA 1994.

24 McKhann G, Drachman D, Folstein M, et al. Clinical diag nosis of Alzheimer's disease: report of the NINCDS ADRDA Work Group under the auspices of Department of Health and Human Services Task Force on Alzheimer's disease. Neurology 1984;34:939-44

25 Hughes CP, Berg L, Danziger WL, et al. A new clinical scale for the staging of dementia. Brf Psychiatry 1982;140:56672 .

26 Folstein MF, Folstein SE, McHugh PR. "Mini-mental state." A practical method for grading the cognitive state of patients for the clinician 7 Psychiatr Res 1975;12:189-98.

27 Wechsler DA. WAIS-R manual. New York: Psychological Corporation, 1981.

28 Mohs RC, Rosen WG, Davis KL. The Alzheimer's disease assessment scale; an instrument for assessing treatment assessment scale; an instrument for assessing
efficacy. Psychopharmacol Bull 1983;19:448-50.

29 Cummings JL, Mega M, Gray K, et al. The neuropsychiatric inventory: comprehensive assessment of psychopathology in dementia. Neurology 1994;44:2308-14.

30 Sano M, Devanand DP, Richards M, et al. A standardized technique for establishing onset and duration of symptoms of Alzheimer's disease. Arch Neurol 1995;52:961-6.
31 Yasuda M, Maeda K, Shimada K, et al. Apolipoprotein E 84 allele and gender difference in risk of Alzheimer's disease. Alzheimer's Research 1995;1:77-81.

32 Wenham PR, Price WH, Blandell G. Apolipoprotein E genotyping by one-stage PCR. Lancet 1991;337:1158-9.

33 Rubin EH. Psychosis in neurologic diseases. Delusions as part of Alzheimer's disease. Neuropsychiatry Neuropsychol Behav Neurol 1992;5:108-13.

34 McKeith IG, Galasko D, Kosaka K, et al. Consensus guidelines for the clinical and pathologic diagnosis of dementia with Lewy bodies (DLB): report of the consortium on DLB international workshop. Neurology 1996;47:1113-24,.

35 Teri L, Larson EB, Reifler BV. Behavioral disturbance in dementia of the Alzheimer's type. If Am Geriatr Soc 1988;36:1-6.

36 Jabeen S, McKeith IG, Fairbairn AF, et al. Psychotic symptoms in Alzheimer's disease. International fournal of Geriatric Psychiatry 1992;7:341-345.

37 Starkstein SE, Vázquez S, Petracca G, et al. A SPECT study of delusions in Alzheimer's disease. Neurology 1994;44: 2055-9.

38 Rosen J, Zubenko GS. Emergence of psychosis and depresion in the longitudinal evaluation of Alzheimer's disease. Biol Psychiatry 1991;29:224-32.

39 Stern Y, Mayeux R, Sano M, et al. Predictors of disease course in patients with probable Alzheimer's disease. Neurology 1987;37:1649-53.

40 Lopez OL, Becker JT, Brenner RP, et al. Alzheimer's disease with delusions and hallucinations: neuropsychological and electroencephalographic correlates. Neurology 1991;41: 906-12.

41 Kotrla KJ, Chacko RC, Harper RG, et al. SPECT findings on psychosis in Alzheimer's disease. Am f Psychiatry 1995; 152:1470-5

42 Lehtovirta $M$, Soininen $H$, Helisalmi S, et al. Clinical and neuropsychological characteristics in familial and sporadic Alzheimer's disease: relation to apolipoprotein E polymorphism. Neurology 1996;46:413-9.

43 Mann DMA, Yates PO, Marcyniuk B. Alzheimer's presenile dementia, senile dementia of Alzheimer type and Down's syndrome in middle age form an age related continuum of pathological changes. Neuropathol Appl Neurobiol 1984;10: 185-207.

44 Nochlin D, Van Belle G, Bird TD, et al. Comparison of the severity of neuropathologic changes in familial and severity of neuropathologic changes in familial and 1993;7:212-22.

45 Förstl H, Burns A, Levy R, et al. Neuropathological correlates of psychotic phenomena in confirmed Alzheimer's disease. Br F Psychiatry 1994;165:53-9.

46 Becker D, Hershkowitz M, Maidler N, et al. Psychopathology and cognitive decline in dementia. $\mathcal{f}$ Nerv Ment Dis 1994;182:701-3.

47 Taylor DC. Factors influencing the occurrence of schizophrenia-like psychosis in patients with temporal lobe epilepsy. Psychol Med 1975;5:249-54.

48 Lopez-Alberola R, Gilchirst D, Barker WW, et al. Analysis of phenotype in Alzheimer's disease (AD) associated with apolipoprotein E (ApoE) alleles [abstract]. Neurology 1994; 44(suppl 2):A207.

49 Lopez OL, Kamboh MI, Becker JT, Kaufer DI, DeKosky $\mathrm{ST}$. The apolipoprotein E $\varepsilon 4$ allele is not associated with psychiatric symptoms or extrapyramidal signs in probable Alzheimer's disease. Neurology 1997;47:794-7. 